\title{
Primeiro registro do ataque de Costalimaita ferruginea em clones de eucalipto em Rondônia
}

\author{
First record of Costalimaita ferruginea attack on Eucalyptus clones in \\ Rondônia, Brazil
}

\author{
Paulo Roberto Magistrali ${ }^{1}$ (1), Jessé Alves Batista ${ }^{2}$ (D), Samuel de Carvalho Andrade ${ }^{3}$ (D), \\ Janice Ferreira do Nascimento ${ }^{4}$ (D), Iris Cristiane Magistrali ${ }^{1}$ (D) \\ ${ }^{1}$ Universidade Federal de Uberlândia - UFU. Monte Carmelo, MG, Brasil \\ ${ }^{2}$ Instituto Federal de Educação, Ciência e Tecnologia de Rondônia - IFRO. Colorado do Oeste, RO, Brasil \\ ${ }^{3}$ Universidade Estadual de Mato Grosso - UNEMAT. Diamantino, MT, Brasil \\ ${ }^{4}$ Instituto Federal de Educação, Ciência e Tecnologia de Rondônia - IFRO. Ji-Paraná, RO, Brasil
}

Como citar: Magistrali, P. R., Batista, J. A., Andrade, S. C., Nascimento, J. F., \& Magistrali, I. C. (2021). Primeiro registro do ataque de Costalimaita ferruginea em clones de eucalipto em Rondônia. Scientia Forestalis, 49(132), e3668. https://doi.org/10.18671/scifor.v49n132.18

\section{Resumo}

A eucaliptocultura brasileira tem aumentado substancialmente nos últimos anos, motivo pelo qual, a incidência de insetos-pragas tem sido mais reportada. Este trabalho relata, pela primeira vez, a ocorrência do Costalimaita ferruginea (Fabricius, 1801) (Coleoptera: Chrysomelidae) em um plantio experimental clonal de Eucalyptus urophylla x Eucalyptus camaldulensis (VM-01) no estado de Rondônia e caracteriza, por meio de análise geoespacial, a distribuição da intensidade do ataque no povoamento. O plantio, instalado no município de ji-Paraná, Rondônia, Brasil, apresentou injúrias nas folhas das árvores aos 8 meses de idade. A intensidade do ataque do inseto-praga nas plantas foi mensurada por meio de notas visuais definidas por: (0) árvores com ausência de ataque (testemunha); (1) desfolhamento menor que o terço superior da copa; (2) desfolhamento do terço superior mais o ponteiro principal atacado e (3) desfolhamento maior que o terço superior da copa mais o ponteiro principal atacado. Todas as árvores foram georreferencias e os mapas da distribuição espacial da intensidade do ataque foram gerados no software Surfer $15^{\circledR}$, a partir do método de interpolação do Inverso do Quadrado da Distância (IQD). Verificou-se que 78\% das árvores apresentaram algum tipo de injúria nas folhas, com predominância de ataque ao terço superior e ao ponteiro principal. Este é o primeiro registro científico do ataque de $C$. ferruginea em um eucaliptal cultivado em Rondônia, indicando que plantios do híbrido VM-01 na Mesorregião do Leste Rondoniense são altamente vulneráveis ao besouro-amarelo-doeucalipto, devendo os silvicultores monitorar e combater futuros surtos para minimizar danos econômicos.

Palavras-chave: análise geoespacial; besouro desfolhador; Eucalyptus urophylla x Eucalyptus camaldulensis; silvicultura de precisão.

\begin{abstract}
The area planted to Eucalyptus sp. has increased substantially recently in Brazil, and for this reason the incidence of insect pests has also increased. This study reports for the first time the occurrence of Costalimaita ferruginea (Fabricius, 1801) (Coleoptera: Chrysomelidae) in an experimental clonal stand Eucalyptus urophylla x Eucalyptus camaldulensis (VM-01) in the state of Rondônia and characterizes, through geospatial analysis, the distribution of the intensity of the attack on the stand. The stand installed in Ji-Paraná, Rondônia, Brazil, presented injuries to the leaves of the trees at 8 months of age. The intensity of insect pest attack on plants was measured using visual notes given as: (0) trees with no attack (control); (1) defoliation less than the upper third of the crown; (2) defoliation of the top third and the upper tip attacked and; (3) defoliation on more than the top third of the crown with the upper tip attacked. All trees were georeferenced and the maps of the spatial distribution of the attack intensity were generated in the Surfer $15^{\circledR}$ software, using the inverse-square-distance (ISD) interpolation method. It was found that $78 \%$ of the trees had some type of injury to the leaves, with a predominance of attack on
\end{abstract}

Fonte de financiamento: Nenhuma.

Conflito de interesse: Nada a declarar.

Autor correspondente: magistralipr@gmail.com

Recebido: 31 janeiro 2021.

Aceito: 4 novembro 2021.

Editor: Mauro Valdir Schumacher.

(c) Este é um artigo publicado em acesso aberto (Open Access) sob a Creative Commons Attribution License, que permite uso, distribuição e Este é um artigo publicado em acesso aberto (Open Access) sob a Creative Commons Attribution Licen
reprodução em qualquer meio, sem restrições desde que o trabalho original seja corretamente citado. 
the upper third and the upper tip. This is the first scientific record of the attack of $C$. ferruginea in eucalypts cultivated in Rondônia, indicating of hybrid VM-01 plantations in the middle region of Eastern Rondonia are highly vulnerable to the eucalyptus yellow beetle, and foresters should monitor and fight future outbreaks to minimize economic damage.

Keywords: geospatial analysis; leaf beetles; Eucalyptus urophylla x Eucalyptus camaldulensis (VM-01); precision forestry.

\section{INTRODUÇÃO}

Os povoamentos florestais no Brasil ocupam 9,0 milhões de hectares e são os mais produtivos do mundo. Com $77 \%$ deste total e produtividade média de $35,3 \mathrm{~m}^{3} \mathrm{ha}^{-1} \mathrm{ano}^{-1}$, a eucaliptocultura, é uma atividade economicamente importante para o país (Indústria Brasileira de Árvores, 2020), sobretudo devido ao rápido crescimento, a adaptabilidade ambiental, e a diversidade de espécies e híbridos que atendem aos diferentes setores da indústria madeireira (Garlet et al., 2009). Neste cenário, o monocultivo do eucalipto (Eucalyptus L'Hér, Myrtaceae) tem aumentado nos últimos anos, inclusive nos estados sem tradição florestal, motivo pelo qual, a ocorrência de insetos-pragas, tais como: formigas cortadeiras, lepidópteros desfolhadores e os coleópteros, têm sido mais frequente (Garlet et al., 2016).

Em relação aos insetos-pragas, os besouros desfolhadores ocupam o terceiro lugar de importância econômica para a silvicultura brasileira, entretanto, novos registros têm aumentado sua relevância para o setor (Montes et al., 2012, Magistrali et al., 2019). A família Chrysomelidae, em especial, compreende um importante grupo de herbívoros, com preferência de ataque para plantas jovens do gênero Eucalyptus (Ohmart \& Edwards, 1991; Arnhold et al., 2019). Popularmente conhecido por "besouro-amarelo" ou "vaquinha", o crisomelídeo Costalimaita ferruginea é um dos principais insetos-pragas que causam danos econômicos, e o quarto mais importante como praga para a cultura do eucalipto (Silva, 1992; Mendes, 2004; Santos et al., 2008; Kassab et al., 2011; Lima et al., 2019). Os adultos deste inseto estão presentes durante toda a fase de desenvolvimento das árvores, ocasionando injúrias típicas de rendilhamento ou perfuramento das folhas, influenciando o crescimento, a sobrevivência e a produtividade dos plantios (Anjos \& Majer, 2003; Xavier et al., 2019).

Buscando minimizar as perdas nos povoamentos, os profissionais do setor florestal têm visto nas análises geoespaciais, uma forma de gerir a produtividade através da silvicultura de precisão (Santos et al., 2017, Cunha Neto et al., 2020), potencializando tratos e métodos silviculturais como o preparo do solo, controle da erosão, desbaste, desrama, adubação e o controle de doenças, pragas e plantas invasoras (Maeda et al., 2014). Análises de variabilidade espacial já aplicadas à agricultura, têm sido uma ferramenta poderosa para monitorar e elaborar mapas da dispersão da entomofauna de interesse, principalmente para programas de manejo integrado de pragas (MIP) que analisam a dependência espacial de dados georreferenciados (Liebhold et al., 1993, Dal Prá et al., 2011, Park et al., 2011). No caso dos programas de proteção florestal, as análises geoespaciais têm detectado as pragas através do monitoramento, sazonal ou contínuo, de pontos de amostragem manuais (Ribeiro et al., 2000) que em muitos casos são de difícil execução em função do comportamento das pragas e das particularidades locais dos plantios (Maeda et al., 2014).

Assim, para que se possa estimar a distribuição espacial dos atributos no campo, um número ideal de pontos amostrais deve ser estabelecido, os quais irão, a partir de modelagem matemática por métodos de interpolação, subsidiar com maior precisão o mapeamento das informações (Souza et al., 2010, Silva et al., 2020). Muitos métodos de interpolação, com diversos níveis de complexidade, estão presentes na literatura, dentre eles, interpoladores estatísticos como a Krigagem e determinísticos como o Inverso do Quadrado da Distância (IQD) têm sido utilizados com maior frequência (Wu et al., 2019; Nogueira et al., 2020). No caso do IQD as médias ponderadas são determinadas univariadamente, ou seja, quanto mais distante um ponto observado estiver de um ponto estimado, menor será seu peso, e por consequência menor será sua influência sobre o valor de inferência (Shukla et al., 2020). Nesse sentido, o presente trabalho objetivou relatar, pela primeira vez, a ocorrência de $C$. ferruginea no estado 
de Rondônia e caracterizar através da análise geoespacial a distribuição da intensidade do ataque causado às árvores de um povoamento experimental clonal do híbrido VM-01 de Eucalyptus urophylla x Eucalyptus camaldulensis, na Mesorregião do Leste Rondoniense.

\section{MATERIAL E MÉTODOS}

Os dados acerca do ataque do besouro foram coletados em outubro de 2019 a partir da realização do censo do povoamento florestal, ou seja, avaliaram-se todas as 154 árvores do plantio clonal de 0,14 ha de E. urophylla $\times$ E. camaldulensis (VM-01), com oito meses de idade, localizado no Instituto Federal de Rondônia - IFRO, no município de Ji-Paraná, Rondônia, Brasil $\left(10^{\circ} 57^{\prime} 30.43^{\prime \prime} \mathrm{S}, 61^{\circ} 54^{\prime} 19.12^{\prime \prime} \mathrm{O}\right)$. No entorno do experimento haviam fragmentos com floresta nativa, áreas com lavoura perene e anual, bem como pastagens com Brachiaria spp.

A região encontra-se no Bioma Amazônico com vegetação do tipo Floresta Ombrófila Aberta (Instituto Brasileiro de Geografia e Estatística, 2004). O clima é do tipo Am (tropicalquente e úmido), com temperatura média anual de $25^{\circ} \mathrm{C}$ e precipitação pluviométrica de $2.500 \mathrm{~mm}$ (Alvares et al., 2013). A identificação do inseto ocorreu através do encaminhamento de 20 exemplares para o Laboratório de Scarabaeoidologia da Universidade Federal do Mato Grosso - UFMT, no município de Cuiabá, Mato Grosso, Brasil. A intensidade de ataque foi mensurada a partir de caracterização visual (Figura 1) das injúrias categorizadas em quatro notas definidas por: (0) árvores com ausência de ataque (testemunha); (1) desfolhamento menor que o terço superior da copa; (2) desfolhamento do terço superior mais o ponteiro principal atacado e (3) desfolhamento maior que o terço superior da copa mais o ponteiro principal atacado, conforme metodologia preconizada por Mendes (2004).

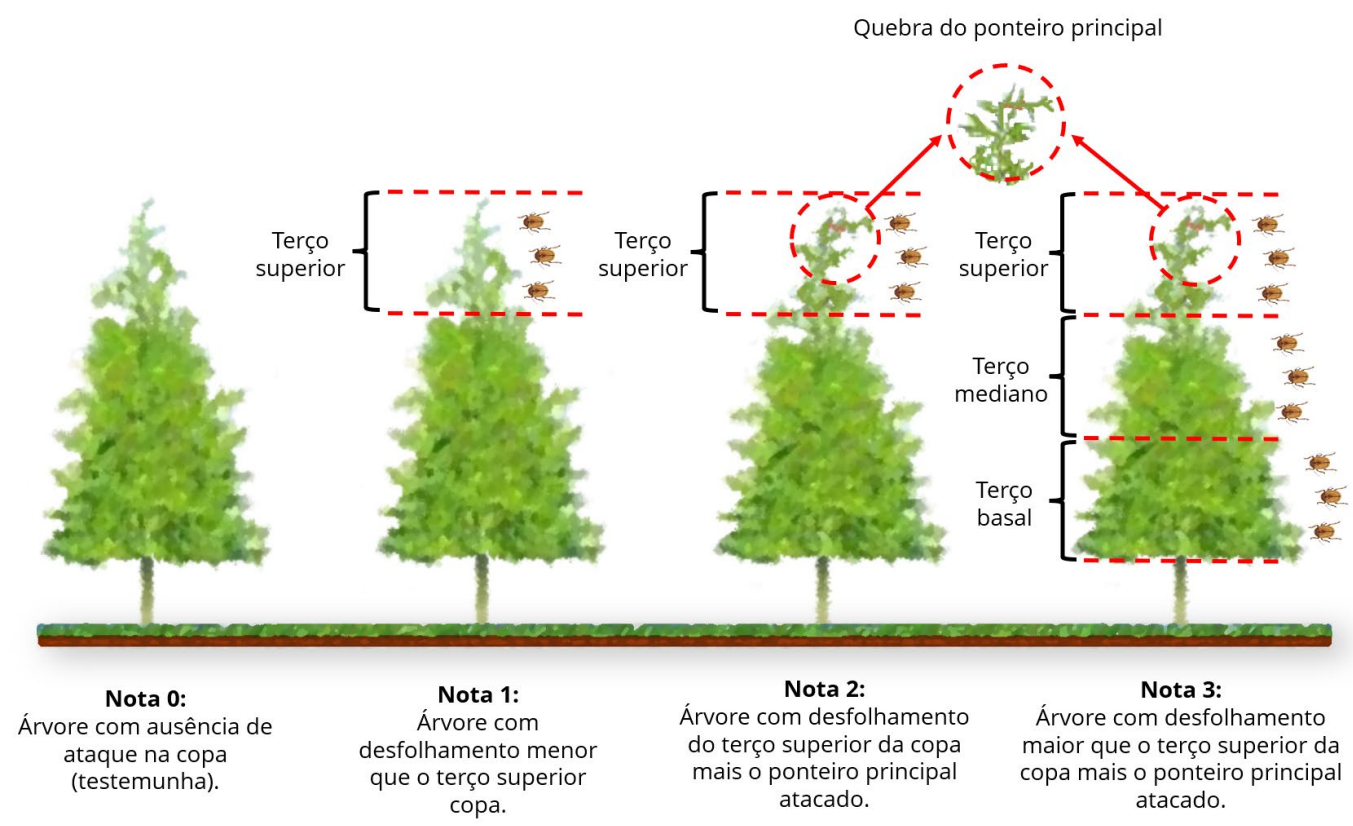

Figura 1. Mensuração da intensidade de ataque do surto de C. ferruginea no plantio de Eucalyptus urophylla x Eucalyptus camaldulensis (VM-01) no município de Ji-Paraná, Rondônia, Brasil. Linhas vermelhas pontilhadas representam as regiões do ataque do inseto-praga.

O grid amostral, com pontos distribuídos $3 \mathrm{~m} \times 3 \mathrm{~m}$, foi estabelecido após a ancoragem sistemática de pontos amostrais junto a todas as árvores do plantio. 0 georreferenciamento dos pontos amostrais foi realizado através de equipamento topográfico equipado com receptor Global Navigation Satellite System - GNSS com precisão de $20 \mathrm{~cm}$. A Figura 2 ilustra a malha amostral georreferenciada bem como a localização geográfica do plantio clonal do híbrido VM-01 no estado de Rondônia. 


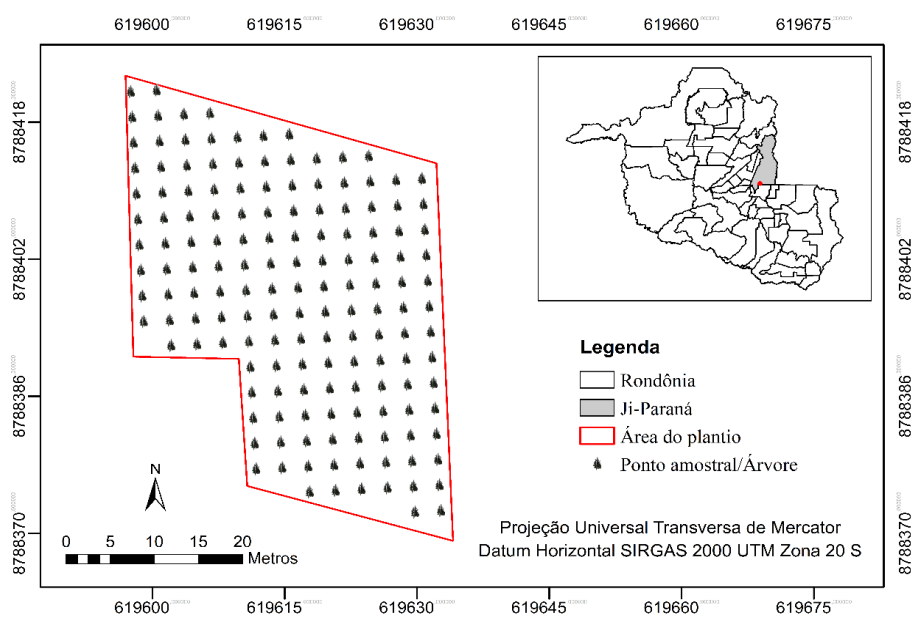

Figura 2. Malha amostral do plantio de Eucalyptus urophylla $\times$ Eucalyptus camaldulensis (VM-01) no município de Ji-Paraná, Rondônia, Brasil.

Os mapas da distribuição espacial do ataque do inseto foram gerados no software Surfer 15 (Golden Software, 2015), a partir do método do IQD (Equação 1) definido por Souza et al. (2010):

$$
\hat{Z}_{i}=\frac{\sum_{i=1}^{n}\left(\frac{1}{d_{i}^{2}} * Z_{i}\right)}{\sum_{i=1}^{n}\left(\frac{1}{d_{i}^{2}}\right)}
$$

Onde:

$\hat{\mathrm{Z}}_{\mathrm{i}}=$ valor interpolado;

$\mathrm{Z}_{\mathrm{i}}=$ valor do atributo amostrado;

$\mathrm{d}_{\mathrm{i}}=$ distância euclidiana entre o ponto amostrado e o estimado.

\section{RESULTADOS E DISCUSSÃO}

Durante a vistoria foram observadas árvores de $E$. urophylla $\times$ E. camaldulensis totalmente atacadas pelo besouro-amarelo-do-eucalipto. O sintoma típico de ataque do inseto são perfurações nas folhas que assumem um aspecto "rendilhado" (Figura 3). Além disso, os insetos adultos podem ocasionar o completo desfolhamento, especialmente em árvores jovens, bem como roer ponteiros e frutificações. O padrão de ataque de $C$. ferruginea no Estado é semelhante aos registrados na literatura (Mendes, 2004; Santos et al., 2008; Dias et al., 2018; Magistrali et al., 2019).
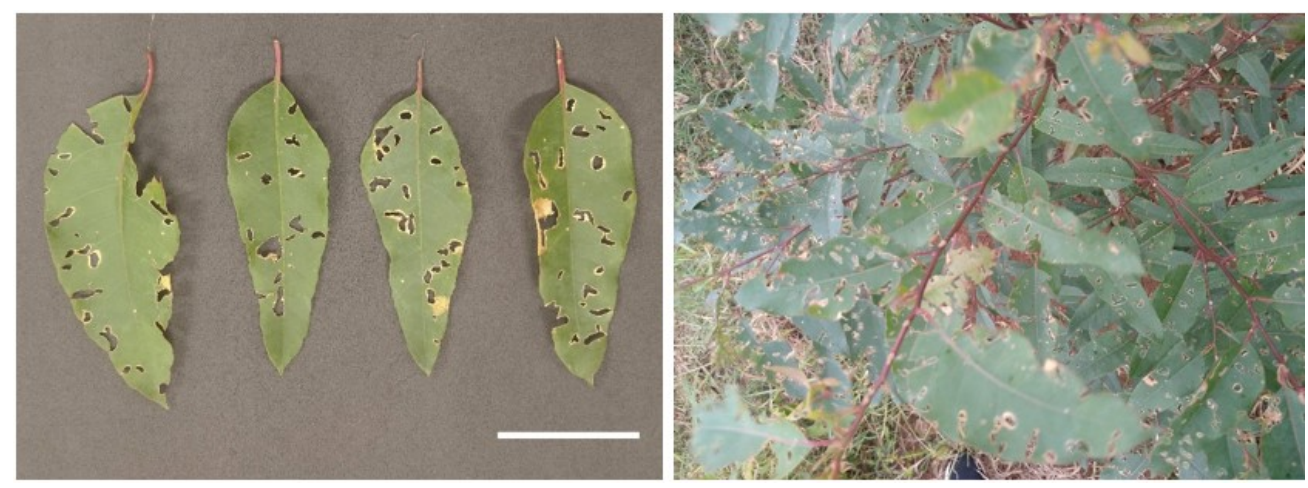

Figura 3. Injúrias observadas nas folhas das árvores do plantio de Eucalyptus urophylla x Eucalyptus camaldulensis (VM-01) no mês de outubro de 2019, no município de Ji-Paraná, Rondônia, Brasil. Barra de escala $=5 \mathrm{~cm}$. 
Os exemplares do inseto foram identificados pelo curador da Seção de Entomologia da Coleção Zoológica (CEMT), Dr. Fernando Zagury Vaz-de-Mello como o crisomelídeo desfolhador Costalimaita ferruginea (Fabricius, 1801) (Coleoptera: Chrysomelidae, Eumolpinae), sendo incorporados ao acervo entomológico da instituição, onde permanecem como material testemunho para futuros estudos do ataque dessa espécie (Figura 4).

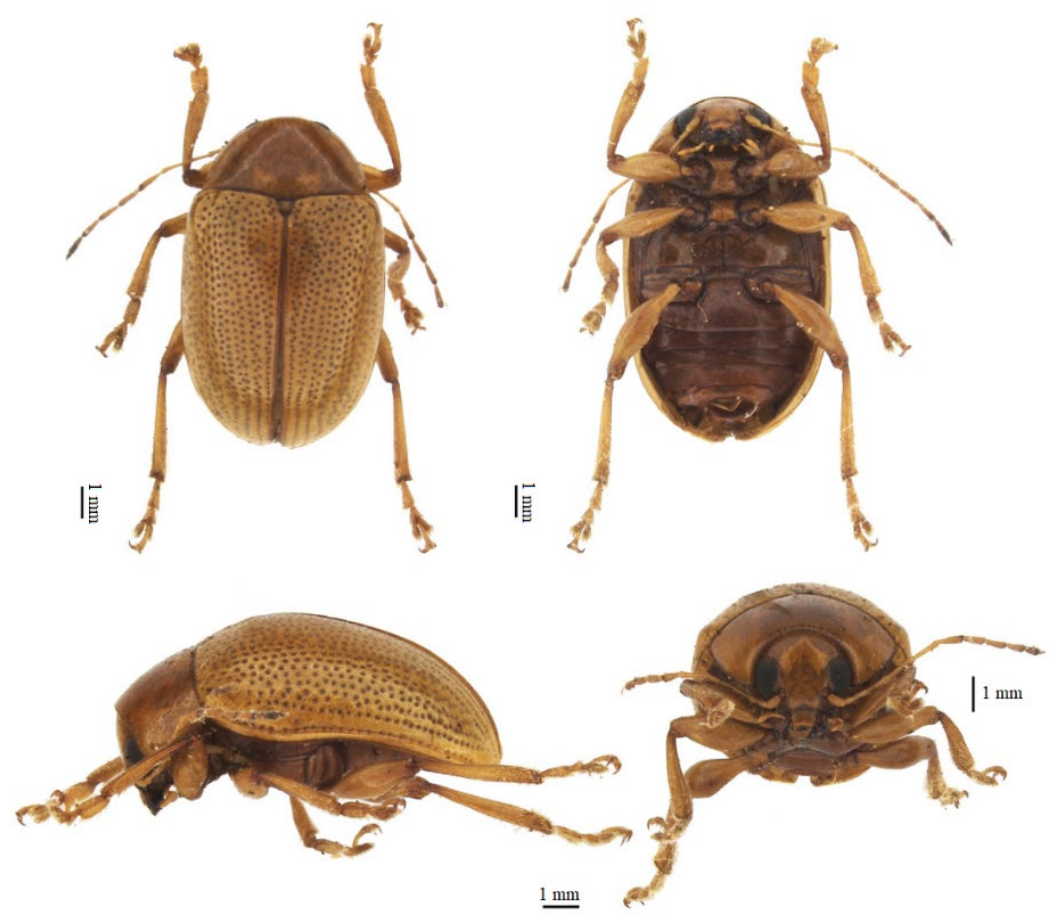

Figura 4. Exemplares de Costalimaita ferruginea depositados no acervo entomológico da Universidade Federal do Mato Grosso - UFMT, Mato Grosso, Brasil.

Relatos da ocorrência do ataque de C. ferruginea em Eucalyptus spp. já foram reportados em diferentes estados brasileiros, assim como é o caso de Minas Gerais (Santos et al., 2008; Pires et al., 2014, Dias et al., 2018, Magistrali et al., 2019), Paraná, Rio Grande do Sul (Santos et al., 2008), Mato Grosso do Sul (Kassab et al., 2011), São Paulo (Montes et al., 2012), Mato Grosso (Pires et al., 2013), Amapá, Pará (Lunz \& Azevedo, 2011), Roraima (Marsaro Junior et al., 2009), Bahia, Espírito Santo (Mafia et al., 2014) e Acre (Santos et al., 2016), Alagoas (Xavier et al., 2019) e Maranhão (Lima et al., 2019).

Todavia, menções do ataque desta espécie para o estado de Rondônia ainda não haviam sido descritas até o momento. Desta maneira, nosso estudo amplia a distribuição geográfica desse crisomelídeo no Brasil, sendo este o primeiro registro desta espécie associada ao híbrido VM-01 de E. urophylla $\times$ E. camaldulensis no Estado e também na Mesorregião do Leste Rondoniense. Uma possível explicação para este fato, pode residir, no histórico de ocupação territorial e na falta de mão de obra especializada para o manejo integrado de pragas nos plantios de eucaliptos do Estado.

Por estar inserido na fronteira agrícola, o agronegócio rondoniense se baseia na expansão da agropecuária (Lobão \& Staduto, 2020), motivo pelo qual, o setor florestal, com cerca de 25 mil ha (Secretaria de Estado da Agricultura, 2020), ainda apresenta uma área inexpressiva se comparado aos milhões de hectares dos plantios florestais presentes na Região Sudeste, Centro-oeste, Nordeste e Sul do Brasil (Indústria Brasileira de Árvores, 2020). Assim, é possível inferir que a ausência de grandes maciços florestais atrelada à falta de interesse em notificar e a carência de conhecimento técnico-científico por parte dos silvicultores, tenham contribuído para a ausência de registros anteriores em Rondônia. 
Ao avaliar a intensidade do surto, através de cenários estimados pelo IQD, verificou-se que $78 \%$ das árvores apresentaram algum tipo de injúria nas folhas (Figura 5A). A distribuição do ataque por classe de injúria demonstrou que $6 \%$ das árvores estavam com desfolhamento menor que o terço superior da copa (Figura 5B), 63\% com desfolhamento do terço superior mais o ponteiro principal atacado (Figura 5C) e 31\% com desfolhamento maior que o terço superior da copa mais o ponteiro principal atacado (Figura 5D). Esses resultados corroboram com Dias et al. (2018) que também registraram ataque deste inseto predominantemente em folhas jovens, nas partes apicais de Eucalyptus grandis e Eucalyptus urophylla.

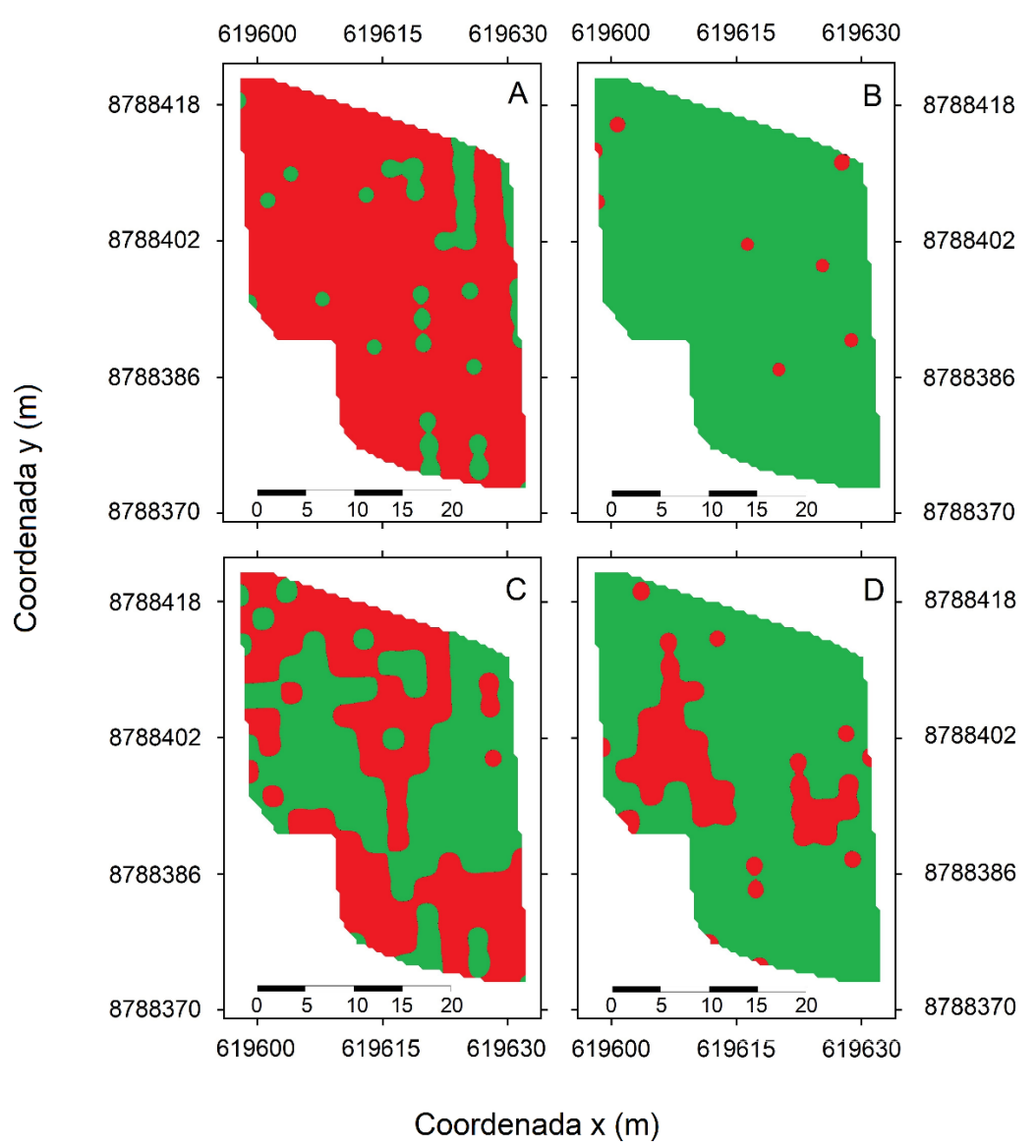

Figura 5. Mapas geoespaciais do surto de C. ferruginea nas diferentes categorias de injúrias observadas nas folhas das árvores do plantio de Eucalyptus urophylla x Eucalyptus camaldulensis (VM-01) no mês de outubro de 2019, no município de Ji-Paraná, Rondônia, Brasil. A - distribuição geral do surto no plantio; $\mathrm{B}$ - árvores com desfolhamento menor que o terço superior da copa; C - árvores com desfolhamento do terço superior mais o ponteiro principal atacado e D - com desfolhamento maior que o terço superior da copa mais o ponteiro principal atacado. - Presença e $\square$ - ausência das injúrias.

Além disso, o plantio clonal do híbrido VM-01 fica localizado próximo a um fragmento florestal de mata nativa, o que pode ter contribuído para o ataque, já que esse inseto também ataca outras mirtáceas como goiabeira (Psidium guajava L.), araçá-do-mato (Psidium guineense Sw.), pitangueira (Eugenia uniflora L.), jambolão (Syzygium cumini Lamarck), por exemplo. No entorno haviam ainda áreas de pastagem bem como de lavouras de milho, o que também pode ter contribuído para a ocorrência do surto, uma vez que as larvas (formas jovens) desse inseto, se desenvolvem no solo e se alimentam das raízes de gramíneas (Anjos \& Majer, 2003; Lunz \& Azevedo, 2011).

O surto, que ocorreu no início da estação chuvosa, pode estar associado ao ciclo biológico do C. ferruginea e, naquelas condições, possivelmente encontrou ambiente adequado para produção de novas gerações. Nesse período, o inseto tende a buscar alimento com maior voracidade (Elek et al., 2000; Mafia et al., 2014; Galli et al., 2017). Associado a isso pode-se 
mencionar o fato de que, em geral, os monocultivos do gênero Eucalyptus tendem a ter menor quantidade de inimigos naturais (predadores, parasitóides e fungos entomopatogênicos) do que fragmentos de mata nativa, favorecendo o aparecimento de altas taxas de populações de insetos-pragas devido a disponibilidade de alimento e a simplificação do ambiente (Xavier et al., 2019).

Neste sentido, relatos de ocorrência do C. ferruginea em plantios jovens de Eucalyptus, quase sempre têm indicado a preferência desta espécie por folhas jovens ou do terço apical da copa das árvores (Anjos \& Majer, 2003, Pires et al., 2013, Santos et al., 2016, Dias et al., 2018), que ao serem rendilhadas, murcham e secam devido à perda de água do limbo foliar (Costa et al., 2011). Em muitos casos os impactos negativos do ataque de C. ferruginea podem ser intensificados, principalmente quando a gema apical das mudas é cortada, induzindo ramificações laterais que atrasam o crescimento inicial do plantio (Montes et al., 2012).

De acordo com Xavier et al. (2019), o efeito do ataque deste crisomelídeo tende a aumentar no decorrer do tempo, uma vez que a uniformidade do desenvolvimento das mudas é reduzida, promovendo o surgimento de plantas suprimidas que produzem menos e causam perdas econômicas. Mendes (2004), ao estudar o efeito da intensidade de ataque do besouro-amarelo em Eucalyptus grandis, com idade de sete a nove meses, relatou perdas na produção da madeira na ordem de 11, 14,4 e 43,6\%, para as árvores atacadas respectivamente nas categorias 1,2 e 3 do sistema de notas adotados em nosso estudo. Além disso, o autor definiu como nível de dano econômico valores de $6 \%$, $3 \%$ e $1 \%$ para as notas 1 , 2 e 3, respectivamente. Para Eucalyptus urophylla e E. urophylla $\times$ E. grandis, localizados em quatro municípios da Mesorregião da Zona da Mata Mineira, as injúrias do ataque do besouroamarelo atingiram percentuais superiores aos descrito pelo autor em relação aos níveis de dano econômico (Magistrali et al., 2019). Resultados semelhantes foram encontrados em nosso estudo, ao utilizarmos a mesma escala de notas, indicando que o $C$. ferruginea pode causar prejuízos financeiros aos futuros plantios de E. urophylla $\times$ E. camaldulensis (VM-01) na região Leste Rondoniense.

Por ser o principal besouro desfolhador capaz de causar prejuízos financeiros ao setor florestal brasileiro (Silva, 1992; Mendes et al., 1998; Anjos \& Majer, 2003; Mendes, 2004), seja pela frequência ou pela periodicidade dos surtos (Magistrali et al., 2019), nossos resultados reforçam a periculosidade do C. ferruginea ao gênero Eucalyptus, servindo de alerta para os silvicultores que pretendem cultivar esta essência florestal em Rondônia. Como destacado anteriormente, devido ao ciclo biológico da espécie, deve-se redobrar os cuidados e o monitoramento, especialmente no início das primeiras chuvas da região. Salienta-se que devido a carência de informações, se faz necessário mais estudos, além de um programa de monitoramento eficiente de $C$. ferruginea a fim de identificar, quais espécies, idades, híbridos e clones de eucaliptos são mais atacados por este inseto-praga.

\section{CONCLUSÃO}

Constatou-se, pela primeira vez, a ocorrência de Costalimaita ferruginea em clones do híbrido Eucalyptus urophylla x Eucalyptus camaldulensis (VM-01) no estado de Rondônia e na Mesorregião do Leste Rondoniense. Em relação as avaliações das injúrias, destaca-se o ataque severo na região do terço superior e da gema apical das copas, com comportamento de predação semelhante aos já descritos na literatura.

\section{AGRADECIMENTOS}

Ao Dr. Fernando Zagury Vaz-de-Mello pelo auxílio na identificação e curadoria dos exemplares do inseto. Ao curso de Pós-Graduação Lato Sensu em Geoprocessamento Ambiental, e o Instituto Federal de Educação, Ciência e Tecnologia de Rondônia - campus Colorado do Oeste e campus ji-Paraná pela capacitação técnica. 


\section{REFERÊNCIAS BIBLIOGRÁFICAS}

Alvares, C. A., Stape, J. L., Sentelhas, P. C., Gonçalves, J. L. M., \& Sparovek, G. (2013). Köppen's climate classification map for Brazil. Meteorologische Zeitschrift (Berlin), 22(6), 711-728. http://dx.doi.org/10.1127/0941-2948/2013/0507.

Anjos, N., \& Majer, J. D. (2003). Leaf-eating Beetles in Brazilian eucalypt plantations. Curtin: School of Environmental Biology.

Arnhold, A., Delabie, J. H. C., \& Gonçalves, D. (2019). External characteristics useful to discriminate the sexes of the beetle Costalimaita lurida (Lefèvre 1891) (Coleoptera: Chrysomelidae, Eumolpinae). Brazilian Journal of Agriculture, 94(1), 84-90. http://dx.doi.org/10.37856/bja.v94i1.3411.

Costa, E. C., D'avila, M., Cantarelli, E. B., Murari, A. B., \& Manzoni, C. G. (2011). Entomologia Florestal. Santa Maria: Ed UFSM.

Cunha Neto, E. M., da Rocha, J. E. C., Bezerra, J. C. F., Melo, M. R. S., \& Alves, G. A. R. (2020). Técnicas de interpolação geoespacial na estimativa do volume de Eucalyptus spp. na mesorregião sudeste paraense. Revista Ibero Americana de Ciências Ambientais, 11(5), 45-54. http://dx.doi.org/10.6008/CBPC2179-6858.2020.005.0005.

Dal Prá, E., Guedes, J. V. C., Cherman, M. A., Jung, A. H., Silva, S. J. P., \& Ribas, G. G. (2011). Uso da geoestatística para caracterização da distribuição espacial de larvas de Diloboderus abderus. Ciência Rural, 41(10), 1689-1694. http://dx.doi.org/10.1590/S0103-84782011001000002.

Dias, T. K. R., Pires, E. M., Souza, A. P., Tanaka, A. A., Monteiro, E. B., \& Wilcken, C. F. (2018). The beetle Costalimaita ferruginea (Coleoptera: Chysomelidae) in Eucalyptus plantations in transition area of Amazon and Cerrado Biomes. Brazilian Journal of Biology = Revista Brasileira de Biologia, 78(1), 47-52. http://dx.doi.org/10.1590/1519-6984.03916.

Elek, J., Bashford, R., \& Candy, S. (2000). Manual for managing leaf beetle defoliation in eucalypt plantations. Australia: Forestry Tasmania.

Galli, J. A., Michelotto, M. D., Fischer, I. H., Soares, M. B. B., \& Martins, A. L. M. (2017). Correlation among damages caused by yellow beetle, climatological elements and production of guava accesses grown in organic system. Revista Brasileira de Fruticultura, 39(3), e-987. http://dx.doi.org/10.1590/010029452017987.

Garlet, J., Costa, E. C., \& Boscardin, J. (2016). Levantamento da entomofauna em plantios de Eucalyptus spp. por meio de armadilha luminosa em São Francisco de Assis - RS. Ciência Florestal, 26(2), 365374. http://dx.doi.org/10.5902/1980509822737.

Garlet, J., Zauza, E. Â. V., Ferreira, F., \& Salvadori, J. R. (2009). Danos provocados por coró-das-pastagens em plantas de eucalipto. Ciência Rural, 39(2), 575-576. http://dx.doi.org/10.1590/S010384782008005000079.

Golden Software. (2015). Surfer for windows. Release 15. Contouring and 3D surface mapping for scientis't engineers. User's Guide. New York: Golden Software, Inc.

Indústria Brasileira de Árvores - IBÁ. (2020). Relatório IBÁ 2020 (122 p.) Recuperado em 28 de janeiro de 2021, de https://iba.org/datafiles/publicacoes/relatorios/relatorio-iba-2020.pdf

Instituto Brasileiro de Geografia e Estatística - IBGE (2004). Mapa da vegetação do Brasil e Mapa de biomas do Brasil. Brasília: IBGE. Recuperado em 28 de janeiro de 2021, de https://www.ibge.gov.br/geociencias/informacoes-ambientais/15842biomas.htmledicao $=16060 \& \mathrm{t}=$ downloads

Kassab, S. O., Mota, T. A., Pereira, F. F., \& Fonseca, P. R. B. (2011). Primero relato de Costalimaita ferruginea (Fabricius, 1801) (Coleoptera: Chrysomelidae) em eucalipto no estado do Mato Grosso do Sul. Ciência Florestal, 21(4), 777-780. http://dx.doi.org/10.5902/198050984522.

Liebhold, A. M., Rossi, R. E., \& Kemp, W. P. (1993). Geostatistic and geographic information system in applied insect ecology. Annual Review of Entomology, 38(1), 303-327. http://dx.doi.org/10.1146/annurev.en.38.010193.001511.

Lima, R. C. A., da Silva, F. F., Freitas, A. S., Almeida, M. F., Batista, M. C., \& Tuelher, E. S. (2019). Registro da incidência de Costalimaita ferruginea em viveiros clonais de eucalipto no estado do Maranhão. Revista Científica Eletrônica de Engenharia Florestal da FAEF, 33(1), 47-53.

Lobão, M. S. P., \& Staduto, J. A. R. (2020). Modernização agrícola na Amazônia brasileira. Revista de Economia e Sociologia Rural, 58(2), 1-18. http://dx.doi.org/10.1590/1806-9479.2020.182276.

Lunz, A. M., \& Azevedo, R. (2011). Caracterização da ocorrência do besouro-amarelo, Costalimaita ferruginea (Fabricius) (Coleoptera: Chrysomelidae), em plantios de eucalipto no Pará (Comunicado técnico, no. 229, 5 p.). Belém: Embrapa Amazônia Oriental. 
Maeda, S., Ahrens, S., Penteado, S. R. C., Oliveira, E. B., Stolle, L., Fowler, J. A. P., \& Bognola, I. A. Silvicultura de precisão. (2014). In A. C. C. Bernardi, J. M. Naime, A. V. Resende, L. H. Bassoi \& R. Y. Inamasu (Eds.), Agricultura de precisão: resultados de um novo olhar (Cap. 47, pp. 467-477). Brasília: Embrapa.

Mafia, R. G., Mendes, J. E. P., \& Corassa, J. N. (2014). Análise comparativa dos surtos e danos causados pelos besouros desfolhadores Costalimaita ferruginea (Fabricius, 1801) e Costalimaita lurida (Lefévre, 1891) (Coleoptera: Chrysomelidae) em plantios de eucalipto. Revista Árvore, 38(5), 829-836. http://dx.doi.org/10.1590/S0100-67622014000500007.

Magistrali, I. C., Souza, R. M., Anjos, N., \& Duarte, C. L. (2019). Besouros crisomelídeos em plantios de eucalipto na Zona da Mata Mineira. Ciência Florestal, 29(1), 425-431. http://dx.doi.org/10.5902/198050984885.

Marsaro Junior, A. L., Bendahan, A. B., \& Medeiros, R. D. (2009). Levantamento de insetos-praga em sistema de integração lavoura-pecuária-floresta em Boa Vista, Roraima (Manual Técnico Embrapa Roraima, Doc. 28, 24 p.). Boa Vista: Embrapa.

Mendes, J. E. P. (2004). Efeitos do ataque de Costalimaita ferruginea (Fabr.) (Coleoptera: Chrysomelidae) sobre o crescimento e produção de Eucalyptus grandis Hill ex Maiden (Tese de doutorado). Universidade Federal de Viçosa, Viçosa.

Mendes, J. E. P., Anjos, N., \& Camargo, F. R. A. (1998). Monitoramento do besouro-amarelo. Folha Florestal, 91, 8-9.

Montes, S. M. N. M., Sato, M. E., Raga, A., \& Cerávolo, L. C. (2012). Avaliação de danos de adultos de Costalimaita ferruginea (Fabricius) (Col.: Chrysomelidae) em Eucalyptus spp. de Presidente Prudente, SP. Arquivos do Instituto Biológico, 79(3), 431-435. http://dx.doi.org/10.1590/S180816572012000300017.

Nogueira, D. B., Silva, A. O., \& Silva, A. P. N. (2020). Comparação entre métodos de interpolação espacial para a estimativa da distribuição de precipitação no Ceará-Brasil. Irriga, 25(1), 131-142. http://dx.doi.org/10.15809/irriga.2020v25n1p131-142.

Ohmart, C. P., \& Edwards, P. B. (1991). Insect herbivory on Eucalyptus. Annual Review of Entomology, 36(1), 637-657. http://dx.doi.org/10.1146/annurev.en.36.010191.003225.

Park, J. J., Lee, J. H., Shin, K. I., Lee, S. E., \& Cho, K. (2011). Geostatistical analysis of the attractive distance of two different sizes of yellow sticky traps for greenhouse whitefly, Trialeurodes vaporariorum (Westwood) (Homoptera: Aleyrodidae), in cherry tomato greenhouses. Australian Journal of Entomology, 50(1), 144-151. http://dx.doi.org/10.1111/j.1440-6055.2010.00796.x.

Pires, E. M., Corassa, J. N., Barreto, M. R., \& Soares, M. A. (2013). New Report of Costalimaita ferruginea (Fabricius) (Coleoptera: Chrysomelidae) on Eucalyptus sp. (Myrtaceae) in Sinop - Mato Grosso, Brazil. EntomoBrasilis, 6(1), 89-90. http://dx.doi.org/10.12741/ebrasilis.v6i1.207.

Pires, E. M., Corassa, J. N., Nogueira, R. M., Oliveira, M. A., Manica, C. L. M., Ferreira, J. A. M., \& Soares, M. A. (2014). New geographical distribution and seasonality of Costalimaita ferruginea (Coleoptera: Chrysomelidae) on Eucalyptus urograndis in Guiricema, Minas Gerais, Brazil. Scientific Electronic Archives, 5(1), 1-4. http://dx.doi.org/10.36560/50201459.

Ribeiro, C. A. A. S., Varella, C. A. A., Sena Junior, D. G., \& Soares, V. P. (2000). Sistemas de Informações Geográficas. In A. Borém, M. P. Giudice, D. M. Queiroz, E. C. Mantovani, L. R. Ferreira, F. X. R. Valle \& R. L. Gomide (Eds.), Agricultura de precisão (pp. 381-407). Viçosa: UFV.

Santos, G. P., Zanuncio, J. C., Zanuncio, T. V., \& Pires, E. M. (2008). Pragas do eucalipto. Informe Agropecuário (Belo Horizonte), 29(1), 43-64.

Santos, M. C., Roveda, M., Zanon, M. L. B., Figueiredo Filho, A., Roik, M., Pacheco, J. M., \& Scavinski, V. (2017). Inventário Florestal Utilizando Técnicas de Silvicultura de Precisão em Povoamentos de Eucalyptus grandis Hill ex Maiden. Floresta e Ambiente, 24(1), e00082714. http://dx.doi.org/10.1590/2179-8087.082714.

Santos, R. S., Gonçalves, R., \& Silva, N. A. (2016). Primeiro registro do besouro-amarelo-do-eucalipto em plantios de eucalipto no Estado do Acre. Revista Ceres, 63(4), 584-587. http://dx.doi.org/10.1590/0034-737X201663040020.

Secretaria de Estado da Agricultura - SEAGRI. (2020). Rondônia avança no cultivo de floresta plantada e deve plantar mais 45 mil hectares nos próximos anos. Recuperado em 22 de janeiro de 2021, de http://www.rondonia.ro.gov.br/rondonia-avanca-no-cultivo-de-floresta-plantada-e-deve-plantarmais-45-mil-hectares-nos-proximos-anos/

Shukla, K., Kumar, P., Mann, G. S., \& Khare, M. (2020). Mapping spatial distribution of particulate matter using Kriging and Inverse Distance Weighting at supersites of megacity Delhi. Sustainable Cities and Society, 54(1), 101997. http://dx.doi.org/10.1016/j.scs.2019.101997. 
Silva, E. R. O., Pereira, M. G., Barros, M. M., Silva, G. O., Vaz, A. F. S., \& Silva Júnior, L. C. (2020). Intensidades amostrais para análise geoestatística da acidez de solo em produção orgânica de banana 'Princesa'. Brazilian Journal of Development, 6(3), 13630-13640. http://dx.doi.org/10.34117/bjdv6n3-286.

Silva, N. A. (1992). Taxonomia, ciclo de vida, dinâmica populacional de Costalimaita ferruginea (Fabr. 1801) (Coleoptera: Chrysomelidae), praga de Eucalyptus spp. (Myrtaceae) (Tese de doutorado). Escola Superior de Agricultura Luiz de Queiroz, Piracicaba.

Souza, G. S., Lima, J. S. S., Xavier, A. C., \& Rocha, W. S. D. (2010). Krigagem ordinária e inverso do quadrado da distância aplicados na espacialização de atributos químicos de um argissolo. Scientia Agraria, 11(1), 73-81. http://dx.doi.org/10.5380/rsa.v11i1.15939.

Wu, C. Y., Mossa, J., Mao, L., \& Almulla, M. (2019). Comparison of different spatial interpolation methods for historical hydrographic data of the lowermost Mississippi River. Annals of GIS, 25(2), 133-151. http://dx.doi.org/10.1080/19475683.2019.1588781.

Xavier, M. E. V., Breda, M. O., Gonzaga, E. P., Santos, D. S., \& Costa, J. G. (2019). Ocorrência e análise de injúrias de Costalimaita ferruginea (Fabricius, 1801) (Coleoptera: Chrysomelidae) em clones de Eucalyptus em Alagoas. Ciência Florestal, 29(3), 1452-1458. http://dx.doi.org/10.5902/1980509835253.

Contribuição dos Autores: PRM: Conceituação, Curadoria de Dados, Investigação, Metodologia, Escrita Primeira Redação, Escrita - Revisão e Edição; JAB: Análise Formal, Metodologia, Supervisão, Escrita - Revisão e Edição; SCA: Análise Formal, Metodologia, Supervisão, Escrita - Revisão e Edição; JFN: Curadoria de Dados, Investigação, Supervisão, Escrita - Revisão e Edição; ICM: Conceituação, Supervisão, Escrita - Revisão e Edição 